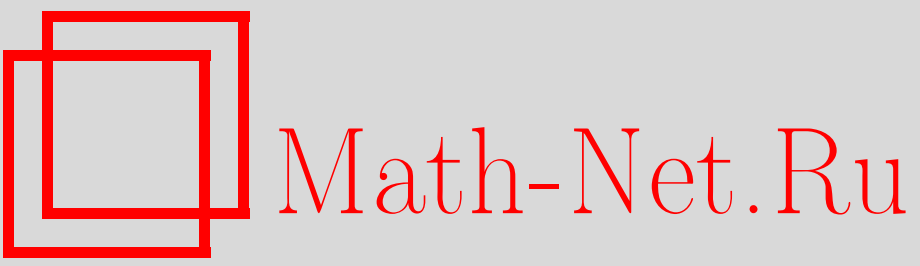

И. Н. Беляева, Н. А. Чеканов, Л. В. Красовская, Н. Н. Чеканова, Компьютерный расчет функций Грина для обыкновенных дифференциальных уравнений третьего порядка, Итоги науки и техн. Сер. Соврем. мат. и ее прил. Темат. обз., 2019, том 165, 3-9

DOI: https://doi.org/10.36535/0233-6723-2019-165-3-9

Использование Общероссийского математического портала Math-Net.Ru подразумевает, что вы прочитали и согласны с пользовательским соглашением

http://www.mathnet.ru/rus/agreement

Параметры загрузки:

IP : 3.93 .64 .190

26 апреля 2023 г., 16:00:04 


\title{
КОМПЬЮТЕРНЫЙ РАСЧЕТ ФУНКЦИЙ ГРИНА ДЛЯ ОБЫКНОВЕННЫХ ДИФФЕРЕНЦИАЛЬНЫХ УРАВНЕНИЙ ТРЕТЬЕГО ПОРЯДКА
}

\author{
(C) 2019 г. И. Н. БЕЛЯЕВА, Н. А. ЧЕКАНОВ,
} Л. В. КРАСОВСКАЯ, Н. Н. ЧЕКАНОВА

\begin{abstract}
АннотАция. В работе представлен способ компьютерного расчета функции Грина в виде обобщенных степенных рядов для линейных дифференциальных уравнений третьего порядка, которые допускают наличие особых регулярных точек. Рассматриваются конкретные краевые задачи, для которых построены функции Грина при помощи разработанной программы.
\end{abstract}

Ключевые слова: обыкновенное дифференциальное уравнение, краевая задача, функция Грина, символьно-численные вычисления, компьютерное моделирование.

\section{COMPUTER CALCULATION OF GREEN'S FUNCTIONS FOR THIRD-ORDER ORDINARY DIFFERENTIAL EQUATIONS}

\author{
(C) 2019 I. N. BELYAEVA, N. A. CHEKANOV,
} L. V. KRASOVSKAYA, N. N. CHEKANOVA

\begin{abstract}
In this paper, we present a method of computer calculation of Green's function in the form of generalized power series for third-order linear differential equations admitting regular singularities. For specific boundary-value problems, we construct Green's functions by using the software proposed.

Keywords and phrases: ordinary differential equation, boundary-value problem, Green function, symbol-numerical computation, computer simulation.
\end{abstract}

AMS Subject Classification: $34 \mathrm{~B} 27$

1. Введение. При решении краевых задач весьма полезна функция Грина, с помощью которой можно, например,

(1) найти решение неоднородного обыкновенного дифференциального уравнения,

(2) вычислить собственные значения и функции при решении задачи на собственные значения для данного дифференциального уравнения,

(3) представить исходную краевую задачу в виде интегрального уравнения Фредгольма и т. д. [1, $3,5,6]$.

Построение функции Грина является актуальной, но достаточно сложной задачей.

Для того чтобы построить функцию Грина, необходимо найти все линейно независимые решения исходной краевой задачи. Таким образом, возникает важная задача интегрирования линейных обыкновенных дифференциальных уравнений, решив которые, можно затем осуществить компьютерный расчет функции Грина краевой задачи для обыкновенного дифференциального 
уравнения. В данной работе решается задача для уравнений третьего порядка с применением компьютерной системы алгебраических вычислений Maple.

2. Вычисление функции Грина для обыкновенных дифференциальных уравнений третьего порядка. Рассмотрим дифференциальное уравнение третьего порядка

$$
p_{3}(x) y^{\prime \prime \prime}+p_{2}(x) y^{\prime \prime}+p_{1}(x) y^{\prime}+p_{0}(x) y=0,
$$

в котором коэффициенты $p_{3}(x), p_{2}(x), p_{1}(x), p_{0}(x)$ являются дважды непрерывно дифференцируемыми функциями на отрезке $[a, b]$. Для уравнения (1) зададим граничные условия

$$
\left\{\begin{array}{l}
\alpha_{1,0} y(a)+\alpha_{1,1} y^{\prime}(a)+\alpha_{1,2} y^{\prime \prime}(a)+\alpha_{1,3} y^{\prime \prime \prime}(a)+\beta_{1,0} y(b)+\beta_{1,1} y^{\prime}(b)+\beta_{1,2} y^{\prime \prime}(b)+\beta_{1,3} y^{\prime \prime \prime}(b)=0, \\
\alpha_{2,0} y(a)+\alpha_{2,1} y^{\prime}(a)+\alpha_{2,2} y^{\prime \prime}(a)+\alpha_{2,3} y^{\prime \prime \prime}(a)+\beta_{2,0} y(b)+\beta_{2,1} y^{\prime}(b)+\beta_{2,2} y^{\prime \prime}(b)+\beta_{2,3} y^{\prime \prime \prime}(b)=0, \\
\alpha_{3,0} y(a)+\alpha_{3,1} y^{\prime}(a)+\alpha_{3,2} y^{\prime \prime}(a)+\alpha_{3,3} y^{\prime \prime \prime}(a)+\beta_{3,0} y(b)+\beta_{3,1} y^{\prime}(b)+\beta_{3,2} y^{\prime \prime}(b)+\beta_{3,3} y^{\prime \prime \prime}(b)=0,
\end{array}\right.
$$

где $\alpha_{i, k}, \beta_{i, k}, i=1,2,3, k=0,1,2,3$, - такие коэффициенты, что

$$
\sum_{i} \sum_{k}\left(\alpha_{i, k}^{2}+\beta_{i, k}^{2}\right) \neq 0
$$

Предположим, что для рассматриваемой задачи (1) с граничными условиями (2) в классе дважды непрерывно дифференцируемых функций существует лишь тривиальное решение. Однако, если для второй производной ослабить требование непрерывности, например, в точке $x=\xi$, $\xi \in[a, b]$, то существует ненулевое решение, которое называется функцией Грина и обозначается $G(x, \xi)$.

Чтобы построить функцию Грина для краевой задачи (1) с условиями (2), необходимо решить сначала задачу Коши в точке $x_{0}$. Будем искать три линейно независимых решения для уравнения (1) в виде разложений (см. [2])

$$
\begin{aligned}
& y_{1}(x)=1+\sum_{k=3}^{N} c_{k}^{(1)}\left(x-x_{0}\right)^{k}, \\
& y_{2}(x)=\left(x-x_{0}\right)+\sum_{k=3}^{N} c_{k}^{(2)}\left(x-x_{0}\right)^{k}, \\
& y_{3}(x)=\left(x-x_{0}\right)^{2} / 2+\sum_{k=3}^{N} c_{k}^{(3)}\left(x-x_{0}\right)^{k},
\end{aligned}
$$

где $c_{k}^{(1)}, c_{k}^{(2)}, c_{k}^{(3)}$ - числовые коэффициенты. Функция Грина имеет следующий вид:

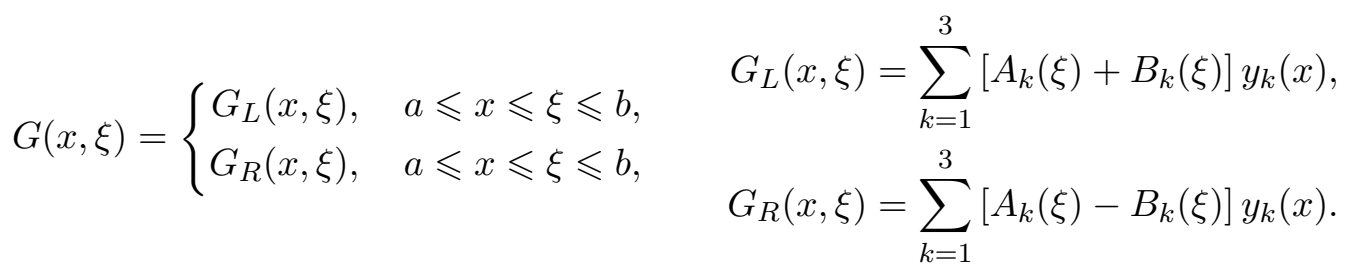

Воспользуемся основными свойствами функции Грина, чтобы найти неизвестные функциональные коэффициенты $A_{k}(\xi), B_{k}(\xi)$.

1. Функция Грина и ее первая производная непрерывны в точке $x=\xi$.

2. Вторая производная функции Грина в точке $x=\xi$ терпит разрыв, который равен

$$
\left.\frac{\partial^{2} G(x, \xi)}{\partial x^{2}}\right|_{x=\xi+0}-\left.\frac{\partial^{2} G(x, \xi)}{\partial x^{2}}\right|_{x=\xi-0}=\frac{1}{p_{3}(\xi)} .
$$

3. Функция Грина удовлетворяет дифференциальному уравнению (1).

4. Функция Грина удовлетворяет граничным условиям (2). 
В теории дифференциальных уравнений (см. $[4,7])$ доказано, что если краевая задача (1) с условиями (2) имеет только тривиальное решение $y(x) \equiv 0$, то существует единственная функция Грина $G(x, \xi)$.

Стоит отметить, что решение задачи Коши ищем в виде степенных рядов; следовательно, и $G(x, \xi)$ будет представлена в виде степенных рядов.

Из свойств 1 и 2 функции Грина получаем систему алгебраических уравнений

$$
\sum_{k=1}^{3} B_{k}(\xi) \cdot y_{k}(\xi)=0, \quad \sum_{k=1}^{3} B_{k}(\xi) \cdot y_{k}^{\prime}(\xi)=0, \quad \sum_{k=1}^{3} B_{k}(\xi) \cdot y_{k}^{\prime \prime}(\xi)=-\frac{1}{2 p_{3}(\xi)} .
$$

Решая систему (6), найдем коэффициенты $B_{k}(\xi), k=1,2,3$. Заметим, что определитель этой системы является вронскианом линейно независимых решений $y_{1}, y_{2}, y_{3}$ :

$$
W(\xi) \equiv W\left[y_{1}, y_{2}, y_{3}\right]=\left|\begin{array}{lll}
y_{1} & y_{2} & y_{3} \\
y_{1}^{\prime} & y_{2}^{\prime} & y_{3}^{\prime} \\
y_{1}^{\prime \prime} & y_{2}^{\prime \prime} & y_{3}^{\prime \prime}
\end{array}\right| \neq 0
$$

Следовательно, система алгебраических уравнений (6) определена и имеет единственное решение $B_{k}(\xi), k=1,2,3$. Далее вычислим функциональные коэффициенты $A_{k}(\xi), k=1,2,3$. Учитывая граничные условия (2), получаем следующую систему трех уравнений:

$$
\begin{aligned}
& A_{1}(\xi)\left[\alpha_{1,0} y_{1}(a)+\alpha_{1,1} y_{1}^{\prime}(a)+\alpha_{1,2} y_{1}^{\prime \prime}(a)+\alpha_{1,3} y_{1}^{\prime \prime \prime}(a)+\beta_{1,0} y_{1}(b)+\beta_{1,1} y_{1}^{\prime}(b)+\beta_{1,2} y_{1}^{\prime \prime}(b)+\beta_{1,3} y_{1}^{\prime \prime \prime}(b)\right]+ \\
& +A_{2}(\xi)\left[\alpha_{1,0} y_{1}(a)+\alpha_{1,1} y_{1}^{\prime}(a)+\alpha_{1,2} y_{1}^{\prime \prime}(a)+\alpha_{1,3} y_{1}^{\prime \prime \prime}(a)+\beta_{1,0} y_{1}(b)+\beta_{1,1} y_{1}^{\prime}(b)+\beta_{1,2} y_{1}^{\prime \prime}(b)+\beta_{1,3} y_{1}^{\prime \prime \prime}(b)\right]+ \\
& +A_{3}(\xi)\left[\alpha_{1,0} y_{1}(a)+\alpha_{1,1} y_{1}^{\prime}(a)+\alpha_{1,2} y_{1}^{\prime \prime}(a)+\alpha_{1,3} y_{1}^{\prime \prime \prime}(a)+\beta_{1,0} y_{1}(b)+\beta_{1,1} y_{1}^{\prime}(b)+\beta_{1,2} y_{1}^{\prime \prime}(b)+\beta_{1,3} y_{1}^{\prime \prime \prime}(b)\right]= \\
& =B_{1}(\xi)\left[\alpha_{1,0} y_{1}(a)+\alpha_{1,1} y_{1}^{\prime}(a)+\alpha_{1,2} y_{1}^{\prime \prime}(a)+\alpha_{1,3} y_{1}^{\prime \prime \prime}(a)-\beta_{1,0} y_{1}(b)-\beta_{1,1} y_{1}^{\prime}(b)-\beta_{1,2} y_{1}^{\prime \prime}(b)-\beta_{1,3} y_{1}^{\prime \prime \prime}(b)\right]+ \\
& +B_{2}(\xi)\left[\alpha_{1,0} y_{1}(a)+\alpha_{1,1} y_{1}^{\prime}(a)+\alpha_{1,2} y_{1}^{\prime \prime}(a)+\alpha_{1,3} y_{1}^{\prime \prime \prime}(a)-\beta_{1,0} y_{1}(b)-\beta_{1,1} y_{1}^{\prime}(b)-\beta_{1,2} y_{1}^{\prime \prime}(b)-\beta_{1,3} y_{1}^{\prime \prime \prime}(b)\right]+ \\
& +B_{3}(\xi)\left[\alpha_{1,0} y_{1}(a)+\alpha_{1,1} y_{1}^{\prime}(a)+\alpha_{1,2} y_{1}^{\prime \prime}(a)+\alpha_{1,3} y_{1}^{\prime \prime \prime}(a)-\beta_{1,0} y_{1}(b)-\beta_{1,1} y_{1}^{\prime}(b)-\beta_{1,2} y_{1}^{\prime \prime}(b)-\beta_{1,3} y_{1}^{\prime \prime \prime}(b)\right] \text {, } \\
& A_{1}(\xi)\left[\alpha_{2,0} y_{1}(a)+\alpha_{2,1} y_{1}^{\prime}(a)+\alpha_{2,2} y_{1}^{\prime \prime}(a)+\alpha_{2,3} y_{1}^{\prime \prime \prime}(a)+\beta_{2,0} y_{1}(b)+\beta_{2,1} y_{1}^{\prime}(b)+\beta_{2,2} y_{1}^{\prime \prime}(b)+\beta_{2,3} y_{1}^{\prime \prime \prime}(b)\right]+ \\
& +A_{2}(\xi)\left[\alpha_{2,0} y_{1}(a)+\alpha_{2,1} y_{1}^{\prime}(a)+\alpha_{2,2} y_{1}^{\prime \prime}(a)+\alpha_{2,3} y_{1}^{\prime \prime \prime}(a)+\beta_{1,0} y_{1}(b)+\beta_{2,1} y_{1}^{\prime}(b)+\beta_{2,2} y_{1}^{\prime \prime}(b)+\beta_{2,3} y_{1}^{\prime \prime \prime}(b)\right]+ \\
& +A_{3}(\xi)\left[\alpha_{2,0} y_{1}(a)+\alpha_{2,1} y_{1}^{\prime}(a)+\alpha_{2,2} y_{1}^{\prime \prime}(a)+\alpha_{2,3} y_{1}^{\prime \prime \prime}(a)+\beta_{2,0} y_{1}(b)+\beta_{2,1} y_{1}^{\prime}(b)+\beta_{2,2} y_{1}^{\prime \prime}(b)+\beta_{2,3} y_{1}^{\prime \prime \prime}(b)\right]= \\
& =B_{1}(\xi)\left[\alpha_{2,0} y_{1}(a)+\alpha_{2,1} y_{1}^{\prime}(a)+\alpha_{2,2} y_{1}^{\prime \prime}(a)+\alpha_{2,3} y_{1}^{\prime \prime \prime}(a)-\beta_{2,0} y_{1}(b)-\beta_{2,1} y_{1}^{\prime}(b)-\beta_{2,2} y_{1}^{\prime \prime}(b)-\beta_{2,3} y_{1}^{\prime \prime \prime}(b)\right]+ \\
& +B_{2}(\xi)\left[\alpha_{2,0} y_{1}(a)+\alpha_{2,1} y_{1}^{\prime}(a)+\alpha_{2,2} y_{1}^{\prime \prime}(a)+\alpha_{2,3} y_{1}^{\prime \prime \prime}(a)-\beta_{2,0} y_{1}(b)-\beta_{2,1} y_{1}^{\prime}(b)-\beta_{2,2} y_{1}^{\prime \prime}(b)-\beta_{2,3} y_{1}^{\prime \prime \prime}(b)\right]+ \\
& +B_{3}(\xi)\left[\alpha_{2,0} y_{1}(a)+\alpha_{2,1} y_{1}^{\prime}(a)+\alpha_{2,2} y_{1}^{\prime \prime}(a)+\alpha_{2,3} y_{1}^{\prime \prime \prime}(a)-\beta_{2,0} y_{1}(b)-\beta_{2,1} y_{1}^{\prime}(b)-\beta_{2,2} y_{1}^{\prime \prime}(b)-\beta_{2,3} y_{1}^{\prime \prime \prime}(b)\right] \text {, } \\
& A_{1}(\xi)\left[\alpha_{3,0} y_{1}(a)+\alpha_{3,1} y_{1}^{\prime}(a)+\alpha_{3,2} y_{1}^{\prime \prime}(a)+\alpha_{3,3} y_{1}^{\prime \prime \prime}(a)+\beta_{3,0} y_{1}(b)+\beta_{3,1} y_{1}^{\prime}(b)+\beta_{3,2} y_{1}^{\prime \prime}(b)+\beta_{3,3} y_{1}^{\prime \prime \prime}(b)\right]+ \\
& +A_{2}(\xi)\left[\alpha_{3,0} y_{1}(a)+\alpha_{3,1} y_{1}^{\prime}(a)+\alpha_{3,2} y_{1}^{\prime \prime}(a)+\alpha_{3,3} y_{1}^{\prime \prime \prime}(a)+\beta_{3,0} y_{1}(b)+\beta_{3,1} y_{1}^{\prime}(b)+\beta_{3,2} y_{1}^{\prime \prime}(b)+\beta_{3,3} y_{1}^{\prime \prime \prime}(b)\right]+ \\
& +A_{3}(\xi)\left[\alpha_{3,0} y_{1}(a)+\alpha_{3,1} y_{1}^{\prime}(a)+\alpha_{3,2} y_{1}^{\prime \prime}(a)+\alpha_{3,3} y_{1}^{\prime \prime \prime}(a)+\beta_{3,0} y_{1}(b)+\beta_{3,1} y_{1}^{\prime}(b)+\beta_{3,2} y_{1}^{\prime \prime}(b)+\beta_{3,3} y_{1}^{\prime \prime \prime}(b)\right]= \\
& =B_{1}(\xi)\left[\alpha_{3,0} y_{1}(a)+\alpha_{3,1} y_{1}^{\prime}(a)+\alpha_{3,2} y_{1}^{\prime \prime}(a)+\alpha_{3,3} y_{1}^{\prime \prime \prime}(a)-\beta_{3,0} y_{1}(b)-\beta_{3,1} y_{1}^{\prime}(b)-\beta_{3,2} y_{1}^{\prime \prime}(b)-\beta_{3,3} y_{1}^{\prime \prime \prime}(b)\right]+ \\
& +B_{2}(\xi)\left[\alpha_{3,0} y_{1}(a)+\alpha_{3,1} y_{1}^{\prime}(a)+\alpha_{3,2} y_{1}^{\prime \prime}(a)+\alpha_{3,3} y_{1}^{\prime \prime \prime}(a)-\beta_{3,0} y_{1}(b)-\beta_{3,1} y_{1}^{\prime}(b)-\beta_{3,2} y_{1}^{\prime \prime}(b)-\beta_{3,3} y_{1}^{\prime \prime \prime}(b)\right]+ \\
& +B_{3}(\xi)\left[\alpha_{3,0} y_{1}(a)+\alpha_{3,1} y_{1}^{\prime}(a)+\alpha_{3,2} y_{1}^{\prime \prime}(a)+\alpha_{3,3} y_{1}^{\prime \prime \prime}(a)-\beta_{3,0} y_{1}(b)-\beta_{3,1} y_{1}^{\prime}(b)-\beta_{3,2} y_{1}^{\prime \prime}(b)-\beta_{3,3} y_{1}^{\prime \prime \prime}(b)\right] \text {. }
\end{aligned}
$$

Полученные функциональные коэффициенты $A_{k}(\xi)$ и $B_{k}(\xi), k=1,2,3$, подставим в формулу (4), в результате чего получим функцию Грина в аналитическом виде.

\section{3. Алгоритм нахождения функции Грина для обыкновенных дифференциальных уравнений третьего порядка. Ввод:}

- $p_{3}(x), p_{2}(x), p_{1}(x), p_{0}(x)$ - функциональные коэффициенты в обыкновенном дифференциальном уравнении третьего порядка (1),

- $N$ - число членов степенного ряда (3),

- $a, b$ - граничные точки заданного отрезка, на котором строится функция Грина $G_{L}(x, \xi)$, 
- $\alpha_{1,0}, \alpha_{1,1}, \alpha_{1,2}, \alpha_{1,3}, \alpha_{2,0}, \alpha_{2,1}, \alpha_{2,2}, \alpha_{2,3}, \alpha_{3,0}, \alpha_{3,1}, \alpha_{3,2}, \alpha_{3,3}, \beta_{1,0}, \beta_{1,1}, \beta_{1,2}, \beta_{1,3}, \beta_{2,0}, \beta_{2,1}, \beta_{2,2}$, $\beta_{2,3}, \beta_{3,0}, \beta_{3,1}, \beta_{3,2}, \beta_{3,3}$ - числовые коэффициенты для краевой задачи в условиях (2).

\section{Вывод:}

- $y_{1}(x), y_{2}(x), y_{3}(x)$ - линейно независимые решения заданного обыкновенного дифференциального уравнения третьего порядка (1),

- $G_{L}(x, \xi)$ - функция Грина на заданном отрезке $[a, b]$, если $a \leqslant x \leqslant \xi \leqslant b$,

- $G_{R}(x, \xi)$ - функция Грина на заданном отрезке $[a, b]$, если $a \leqslant \xi \leqslant x \leqslant b$.

\section{Описание шагов алгоритма:}

1. Процедура построения трех линейно независимых решений дифференциального уравнения третьего порядка в виде обобщенных степенных рядов (см. [2]).

2. Проверка найденных решений дифференциального уравнения.

3. Решение системы алгебраических уравнений (6) для вычисления функциональных коэффициентов $B_{k}(\xi), k=1,2,3$.

4. Проверка найденных функциональных коэффициентов $B_{k}(\xi), k=1,2,3$.

5. Решение системы алгебраических уравнений (8), (9), (10) для вычисления коэффициентов $A_{k}(\xi), k=1,2,3$.

6. Проверка найденных коэффициентов $A_{k}(\xi), k=1,2,3$.

7. Построение функции Грина $G_{L}(x, \xi)$ при условии $a \leqslant x \leqslant \xi \leqslant b$ по формуле (4).

8. Построение функции Грина $G_{R}(x, \xi)$ при условии $a \leqslant \xi \leqslant x \leqslant b$ по формуле 4.

9. Проверка основных четырех свойств функции Грина $G(x, \xi)$.

4. Компьютерный расчет функции Грина. Для тестирования разработанной программы было рассмотрено дифференциальное уравнение

$$
y^{\prime \prime \prime}-6 y^{\prime \prime}+11 y^{\prime}-6 y=0
$$

с граничными условиями

$$
y(0)=0, \quad y(1)=0, \quad y^{\prime \prime}(0)=0 .
$$

Сначала вычислим функцию Грина для данной краевой задачи без использования ЭВМ. Фундаментальная система решений уравнения (11) с условиями (12) имеет вид

$$
y_{1}=e^{x}, \quad y_{2}=e^{2 x}, \quad y_{2}=e^{3 x} .
$$

Функция $G(x, \xi)$ может быть построена из решений (13) по рассмотренному выше способу. Учитывая свойства функции Грина 1 и 2 , получаем систему

$$
\left\{\begin{array}{l}
B_{1}(\xi) \cdot y_{1}(\xi)+B_{2}(\xi) \cdot y_{2}(\xi)+B_{3}(\xi) \cdot y_{3}(\xi)=0 \\
B_{1}(\xi) \cdot y_{1}^{\prime}(\xi)+B_{2}(\xi) \cdot y_{2}^{\prime}(\xi)+B_{3}(\xi) \cdot y_{3}^{\prime}(\xi)=0 \\
B_{1}(\xi) \cdot y_{1}^{\prime \prime}(\xi)+B_{2}(\xi) \cdot y_{2}^{\prime \prime}(\xi)+B_{3}(\xi) \cdot y_{3}^{\prime \prime}(\xi)=-\frac{1}{2 p_{3}(\xi)}
\end{array}\right.
$$

Поскольку $p_{3}(\xi) \neq 0$, система уравнений (14) всегда разрешима и имеет единственное решение. Таким образом, главный определитель данной системы $(14)$ - это вронскиан $W\left[y_{1}, y_{2}, y_{3}\right] \neq 0$. Решая систему уравнений (14)) находим коэффициенты $B_{k}(\xi), k=1,2,3$ :

$$
B_{1}(\xi)=-\frac{1}{4} e^{-\xi}, \quad B_{2}(\xi)=\frac{1}{2} e^{-2 \xi}, \quad B_{3}(\xi)=-\frac{1}{4} e^{-3 \xi} .
$$

Далее, учитывая граничные условия (12), получим систему уравнений

$$
\left\{\begin{aligned}
A_{1}(\xi)+A_{2}(\xi)+A_{3}(\xi) & =-B_{1}(\xi)-B_{2}(\xi)-B_{3}(\xi), \\
A_{1}(\xi)+4 A_{2}(\xi)+9 A_{3}(\xi) & =-B_{1}(\xi)-4 B_{2}(\xi)-9 B_{3}(\xi), \\
A_{1}(\xi)+e A_{2}(\xi)+e^{2} A_{3}(\xi) & =B_{1}(\xi)+e B_{2}(\xi)+e^{2} B_{3}(\xi),
\end{aligned}\right.
$$


решая которую, найдем коэффициенты $A_{k}(\xi), k=1,2,3$ :

$$
\begin{aligned}
& A_{1}(\xi)=-\frac{1}{4\left(3 e^{2}-8 e+5\right)}\left(-20 e^{1+\xi}+5 e^{2 \xi}+10 e^{2}+8 e^{1-2 \xi}-3 e^{2+2 \xi}\right) e^{-3 \xi}, \\
& A_{2}(\xi)=\frac{1}{2\left(3 e^{2}-8 e+5\right)}\left(-8 e^{1+\xi}+8 e^{2 \xi}+8 e^{2}-5 e^{\xi}-3 e^{2+\xi}\right) e^{-3 \xi} \\
& A_{3}(\xi)=-\frac{1}{4\left(3 e^{2}-8 e+5\right)}\left(-12 e^{1+\xi}-5+6 e^{2 \xi}+3 e^{2}+8 e\right) e^{-3 \xi}
\end{aligned}
$$

Подставляя найденные функции $B_{k}(\xi), A_{k}(\xi), k=1,2,3$, в (4), вычисляем точную функцию Грина для задачи (11) с условиями (12) в виде

$$
\begin{gathered}
G(x, \xi)= \begin{cases}G_{L}(x, \xi), & 0 \leqslant x \leqslant \xi \leqslant 1, \\
G_{R}(x, \xi), & 0 \leqslant x \leqslant \xi \leqslant 1,\end{cases} \\
G_{L}(x, \xi)=\frac{e^{x}\left(-5 / 2 e^{-\xi}+5 e^{1-2 \xi}-5 / 2 e^{2-3 \xi}\right)}{3 e^{2}-8 e+5}+\frac{e^{2 x}\left(4 e^{-\xi}-8 e^{1-2 \xi}+4 e^{2-3 \xi}\right)}{3 e^{2}-8 e+5}+ \\
+\frac{e^{3 x}\left(-3 / 2 e^{-\xi}+3 e^{1-2 \xi}-3 / 2 e^{2-3 \xi}\right)}{3 e^{2}-8 e+5}, \\
G_{R}(x, \xi)=\frac{e^{x}\left(3 / 2 e^{2-\xi}-4 e^{1-\xi}+5 e^{1-2 \xi}-5 / 2 e^{2-3 \xi}\right)}{3 e^{2}-8 e+5}+\frac{e^{2 x}\left(4 e^{-\xi}-3 e^{2-2 \xi}-5 e^{-2 \xi}+4^{2-3 \xi}\right)}{3 e^{2}-8 e+5}+ \\
+\frac{e^{3 x}\left(-3 / 2 e^{-\xi}+3 e^{1-2 \xi}+5 / 2 e^{-3 \xi}-4 e^{1-3 \xi}\right)}{3 e^{2}-8 e+5} .
\end{gathered}
$$

С помощью разработанной программы в среде Maple для краевой задачи (11) с условиями (12) была вычислена приближенная функция Грина, в которой приведем первые шесть членов $N=6$ :

$$
\begin{aligned}
& G(x, \xi)=\left\{\begin{array}{l}
G_{L}^{6}(x, \xi), \quad 0 \leqslant x \leqslant \xi \leqslant 1, \\
G_{R}^{6}(x, \xi), \quad 0 \leqslant x \leqslant \xi \leqslant 1,
\end{array}\right. \\
& G_{L}^{6}(x, \xi)= \frac{19663}{35489} x-\frac{82489}{35489} x \xi+\frac{308167}{70978} x \xi^{2}-\frac{216293}{212934} x^{3}-\frac{1059601}{212934} x \xi^{3}+\frac{907379}{212934} x^{3} \xi-\frac{98315}{70978} x^{4}+ \\
&+ \frac{3462703}{851736} x \xi^{4}-\frac{3389837}{425868} x^{3} \xi^{2}+\frac{412445}{70978} x^{4} \xi-\frac{4699457}{4258680} x^{5}-\frac{10969609}{4258680} x \xi^{5}+\frac{11655611}{1277604} x^{3} \xi^{3}- \\
&- \frac{1540835}{141956} x^{4} \xi^{2}+\frac{19714871}{4258680} x^{5} \xi-\frac{137641}{212934} x^{6}+\frac{46783051}{12776040} x \xi^{6}-\frac{38089733}{5110416} x^{3} \xi^{4}+ \\
&+ \frac{5298005}{425868} x^{4} \xi^{3}-\frac{73651913}{8517360} x^{5} \xi^{2}+\frac{577423}{212934} x^{6} \xi+\frac{120665699}{25552080} x^{3} \xi^{5}-\frac{17313515}{1703472} x^{4} \xi^{4}+ \\
&+ \frac{253244639}{25552080} x^{5} \xi^{3}-\frac{2157169}{425868} x^{6} \xi^{2}-\frac{514613561}{76656240} x^{3} \xi^{6}+\frac{10969609}{1703472} x^{2} \xi^{5}-\frac{827586017}{102208320} x^{5} \xi^{4}+ \\
&+ \frac{7417207}{1277604} x^{6} \xi^{3}-\frac{46783051}{5110416} x^{4} \xi^{6}+\frac{2621736551}{511041600} x^{5} \xi^{5}-\frac{24238921}{5110416} x^{6} \xi^{4}-\frac{11181149189}{1533124800} x^{5} \xi^{6}+ \\
&+ \frac{76787263}{25552080} x^{6} \xi^{5}-\frac{327481357}{76656240} x^{6} \xi^{6}, \\
& G_{R}^{6}(x, \xi)= \frac{19663}{35489} x+\frac{1}{2} \xi^{2}-\frac{117978}{35489} x \xi+\frac{1}{2} x^{2}-\xi^{3}+\frac{521101}{70978} x \xi^{2}-3 x^{2} \xi-\frac{3359}{212934} x^{3}-\frac{25}{24} \xi^{4}+ \\
&+\frac{324471}{35489} x \xi^{3}+\frac{25}{4} x^{2} \xi^{2}+\frac{3359}{35489} x^{3} \xi-\frac{292555}{851736} x^{4}-\frac{3}{4} \xi^{5}+\frac{6656713}{851736} x \xi^{4}-\frac{15}{2} x^{2} \xi^{3}- \\
&-\frac{195827}{425868} x^{3} \xi^{2}+\frac{292555}{141956} x^{4} \xi-\frac{1505447}{4258680} x^{5}+\frac{301}{720} \xi^{6}-\frac{3608633}{709780} x \xi^{5}+\frac{301}{48} x^{2} \xi^{4}+ \\
&+\frac{54079}{70978} x^{3} \xi^{3}-\frac{7807831}{1703472} x^{4} \xi^{2}+\frac{1505447}{709780} x^{5} \xi-\frac{5834731}{25552080} x^{6}+\frac{31962119}{6388020} x \xi^{6}-
\end{aligned}
$$




$$
\begin{aligned}
& -\frac{161}{40} x^{2} \xi^{5}-\frac{3807359}{5110416} x^{3} \xi^{4}+\frac{1627427}{283912} x^{4} \xi^{3}-\frac{39369539}{8517360} x^{5} \xi^{2}-\frac{5834731}{4258680} x^{6} \xi+\frac{605}{144} x^{2} \xi^{6}+ \\
& +\frac{2218579}{4258680} x^{3} \xi^{5}-\frac{100407955}{20441664} x^{4} \xi^{4}+\frac{8105023}{1419560} x^{5} \xi^{3}-\frac{30301211}{10220832} x^{6} \xi^{2}-\frac{26994701}{76656240} x^{3} \xi^{6}
\end{aligned}
$$

Было проведено сравнение вычисленной с помощью разработанной программы функции Грина (19) с точной функцией, найденной по формуле (18). Проведенные расчеты показывают, что полученная приближенная функция Грина $G_{\text {calc }}$ отличается на $1.3 \%$ от точной функции Грина $G_{t h e o r}$, при этом учитывается число членов ряда до $N=13$. Если же число членов ряда увеличить, то относительная погрешность уменьшается. Так, при числе членов степенного ряда $N=16$ относительная погрешность становится равной $0.003 \%$.

Стоит также заметить, что погрешность зависит и от $\xi$. Проведенные расчеты при числе членов ряда $N=13$ показывают, что абсолютная погрешность $\Delta=\left|G_{\text {theor }}-G_{\text {calc }}\right|$ при $\xi=0.25$ равна $\Delta=8 \cdot 10^{-7}$, при $\xi=0.5$ имеем $\Delta=1.5 \cdot 10^{-6}$, при $\xi=0.75$ получаем $\Delta=0.00015$. Относительная погрешность $\varepsilon=\Delta / G_{\text {theor }} \cdot 100 \%$ при $\xi=0.25$ равна $\varepsilon=0.038 \%$. Если $\xi=0.5$, то $\varepsilon=1.3 \%$. Если $\xi=0.75$, то $\varepsilon=3 \%$.

Была также вычислена абсолютная погрешность при числе членов ряда $N=16$. В этом случае при $\xi=0.25$ получаем $\Delta=6 \cdot 10^{-9}$, при $\xi=0.5$ имеем $\Delta=8 \cdot 10^{-9}$, при $\xi=0.75$ получаем $\Delta=6 \cdot 10^{-7}$. Относительная погрешность при $\xi=0.25$ равна $\varepsilon=4 \cdot 10^{-5} \%$, при $\xi=0.5$ получаем $\varepsilon=0.003 \%$, при $\xi=0.75$ имеем $\varepsilon=0.006 \%$.

Рассмотрим дифференциальное уравнение

$$
y^{\prime \prime \prime}=0
$$

с граничными условиями

$$
y(0)=0, \quad y(1)=0, \quad y^{\prime}(0)=y^{\prime}(1) .
$$

Используя разработанную программу, получаем функцию Грина

$$
G(x, \xi)= \begin{cases}G_{L}(x, \xi), & 0 \leqslant x \leqslant \xi \leqslant 1, \\ G_{R}(x, \xi), & 0 \leqslant x \leqslant \xi \leqslant 1,\end{cases}
$$

где

$$
G_{L}=\frac{1}{2}\left(x^{2} \xi-x \xi^{2}-x^{2}+x \xi\right), \quad G_{R}=\frac{1}{2}\left(x^{2} \xi-x \xi^{2}-x \xi+\xi^{2}\right) .
$$

Полученное выражение (22) совпадает с точным выражением и является антисамосопряженным (см. $[5,6])$.

Рассмотрим дифференциальное уравнение (20), но с граничными условиями

$$
y(0)=0, \quad y(1)=0, \quad y^{\prime}(0)+y^{\prime}(1)=0 .
$$

Данное уравнение имеет нетривиальное решение; следовательно, функции $G(x, \xi)$ не существует.

Далее, рассмотрим дифференциальное уравнение (20) с граничными условиями

$$
y(0)=0, \quad y^{\prime}(1)=0, \quad y^{\prime}(0)=y(1) .
$$

Используя разработанную программу, получаем функцию Грина:

$$
G(x, \xi)= \begin{cases}G_{L}(x, \xi), & 0 \leqslant x \leqslant \xi \leqslant 1, \\ G_{R}(x, \xi), & 0 \leqslant x \leqslant \xi \leqslant 1,\end{cases}
$$

где

$$
G_{L}=-\frac{1}{2} x^{2} \xi^{2}+x^{2} \xi+x \xi^{2}-\frac{1}{2} x^{2}-x \xi, \quad G_{R}=-\frac{1}{2} x^{2} \xi^{2}+x^{2} \xi+x \xi^{2}-2 x \xi+\frac{1}{2} \xi^{2} .
$$

Стоит отметить, что в данном случае, функция Грина $G(x, \xi)$, заданная формулой $(25)$, не является антисамосопряженной.

Представленные краевые задачи показывают важную роль граничных условий.

Рассмотрим дифференциальное уравнение

$$
y^{\prime \prime \prime}-x^{2} y^{\prime \prime}+(a+b-1) x y^{\prime}-a b y=0
$$


где $a, b$ - параметры, с граничными условиями

$$
y(0)=0, \quad y(1)=0, \quad y^{\prime}(0)=y^{\prime}(1) .
$$

Используя разработанную программу, вычисляем функцию Грина в виде степенного ряда до $N=5$. Для простоты положим значения параметров равными $a=b=1$; тогда функция Грина имеет вид

$$
\begin{gathered}
G(x, \xi)= \begin{cases}G_{L}(x, \xi), & 0 \leqslant x \leqslant \xi \leqslant 1, \\
G_{R}(x, \xi), & 0 \leqslant x \leqslant \xi \leqslant 1,\end{cases} \\
G_{L}=-\frac{1}{120} x^{5}+\frac{1}{3} x^{2} \xi^{3}-\frac{24}{125} x \xi^{4}-\frac{3}{25} x^{2} \xi^{2}+\frac{12}{25} x^{2} \xi-\frac{173}{375} x \xi^{2}-\frac{1}{2} x^{2}+\frac{64}{125} x \xi \\
G_{R}=\frac{1}{12} x^{3} \xi^{2}+\frac{183}{1000} x \xi^{4}-\frac{2}{15} \xi^{5}-\frac{3}{25} x^{2} \xi^{2}+\frac{12}{25} x^{2} \xi-\frac{173}{375} x \xi^{2}-\frac{61}{125} x \xi+\frac{1}{2} \xi^{2} .
\end{gathered}
$$

5. Заключение. В работе предложен способ символьно-численного расчета функции Грина в виде степенных рядов для обыкновенных дифференциальных уравнений третьего порядка. Проведено тестирование разработанной программы. Выполнен компьютерный расчет решения конкретных краевых задач.

\section{СПИСОК ЛИТЕРАТУРЫ}

1. Айнс Э. Л. Обыкновенные дифференциальные уравнения. - Харьков: ОНТИ, 1939.

2. Беляева И. Н., Чеканов Н. А. Символьно-численное интегрирование линейного дифференциального уравнения третьего порядка в виде обобщенных рядов/ Свидетельство о государственной регистрации программы для ЭВМ № 2010614257 - 2010.

3. Камке Э. Справочник по обыкновенным дифференциальным уравнениям. - М.: Наука, 1965.

4. Краснов М. Л., Киселев А. И., Макаренко Г. И. Интегральные уравнения. - М.: Наука, 1976.

5. Сансоне Дж. Обыкновенные дифференциальные уравнения. Т. 1. - М.: ИЛ, 1953.

6. Сансоне Дж. Обыкновенные дифференциальные уравнения. Т. 2. - М.: ИЛ, 1954.

7. Трикоми Ф. Дифференциальные уравнения. - М.: ИЛ, 1962.

Беляева Ирина Николаевна

Белгородский государственный национальный исследовательский университет

E-mail: ibelyaeva@bsu.edu.ru

Чеканов Николай Александрович

Белгородский государственный национальный исследовательский университет

E-mail: Chekanov@bsu.edu.ru

Красовская Людмила Владимировна

Белгородский государственный национальный исследовательский университет

E-mail: krasovskaya@bsu.edu.ru

Чеканова Наталья Николаевна

Харьковский учебно-научный институт

государственного высшего учебного заведения «Университет банковского дела»

E-mail: chekanova76@list.ru 